\title{
Accentuated Skin Markings: A Clinical Clue for Idiopathic Eruptive Macular Pigmentation
}

\author{
${ }^{1}$ Stuti Khare, ${ }^{2}$ Bhushan Madke, ${ }^{3}$ Adarshlata Singh
}

\begin{abstract}
Idiopathic eruptive macular pigmentation (IEMP) is a distinct clinicopathologic entity of which less cases have been reported in the literature. We report here a case of a 16 -year-old boy who had completely asymptomatic brownish-colored lesions distributed over the trunk, back, and upper limbs since 4 months, and histopathologic findings showed features consistent with diagnosis of IEMP with one additional finding having papillomatosis with moderate acanthosis. We are reporting this case with additional clinical finding that is accentuated skin markings similar to acanthosis on dermoscopy, which aid in clinical diagnosis of this less reported entity. This helps avoidance of unnecessary treatment for this self-resolving state.
\end{abstract}

Keywords: Dermoscopy, Differentials, Idiopathic eruptive macular pigmentation, Pigmented papillomatosis.

How to cite this article: Khare S, Madke B, Singh A. Accentuated Skin Markings: A Clinical Clue for Idiopathic Eruptive Macular Pigmentation. Int J Recent Surg Med Sci 2016;2(2):108-110.

\section{Sources of support: Nil}

Conflict of interest: None

\section{INTRODUCTION}

Idiopathic eruptive macular pigmentation (IEMP) was first described by Degos et al. ${ }^{1}$ The disease occurs mainly during childhood and adolescence. The etiology is still unknown, and lesions usually appear abruptly and gradually disappear within a few months. The condition is characterized by previous absence of erythema and pruritus. The diagnostic criteria for approaching the disease was given by Galdeano et al. ${ }^{2}$

- Eruption of brownish nonconfluent asymptomatic macule involving the trunk, neck, and proximal extremities in children or adolescents

- Absence of a preceding inflammatory process.

\footnotetext{
${ }^{1}$ Resident, ${ }^{2}$ Associate Professor, ${ }^{3}$ Professor and Head

${ }^{1}$ Department of Dermatology, Datta Meghe Institute of Medical Sciences, Wardha, Maharashtra, India

${ }^{2,3}$ Department of Dermatology, Acharya Vinoba Bhave Rural Hospital, Wardha, Maharashtra, India

Corresponding Author: Stuti Khare, Resident, Department of Dermatology, Datta Meghe Institute of Medical Sciences Wardha, Maharashtra, India, Phone: +918605607566, e-mail: stuti109@gmail.com
}

- No previous drug exposure

- Basal cell layer hyperpigmentation of the epidermis and prominent dermal melanophages without visible basal layer damage or lichenoid inflammatory infiltrate

- Normal mast cell count.

\section{CASE REPORT}

A 16-year-old male presented to our outpatient department with multiple hyperpigmented skin lesions over trunk and back that appeared insidiously. There was no history of preceding symptoms of itching and erythema. Patient denied any history of previous drug consumption. There was no history of similar skin lesions in the past, and no other family member had similar skin eruptions in the recent past.

On cutaneous examination, multiple well-defined nonconfluent brown-colored round-to-oval macules and papules of varying sizes were present on the trunk, back, and upper limbs (Figs 1 and 2). Most of the pigmented lesions showed accentuation of skin markings. Mucous membranes, palms, and soles were spared. Routine hematological investigations were within normal range. Dermoscopy of the lesion showed accentuated darkly pigmented skin markings similar to that seen in acanthosis nigricans (Fig. 3). We made a provisional diagnosis of IEMP, lichen planus pigmentosus and post inflammatory pigmentation.

Skin biopsy showed basket weave hyperkeratosis and papillomatosis with moderate acanthosis and increased

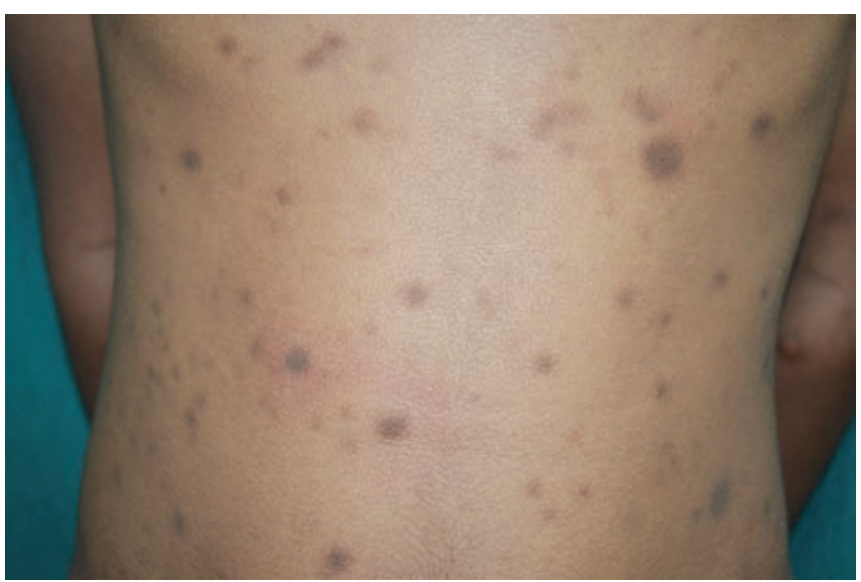

Fig. 1: Trunk showing multiple hyperpigmented macules, papules, and small plaques 


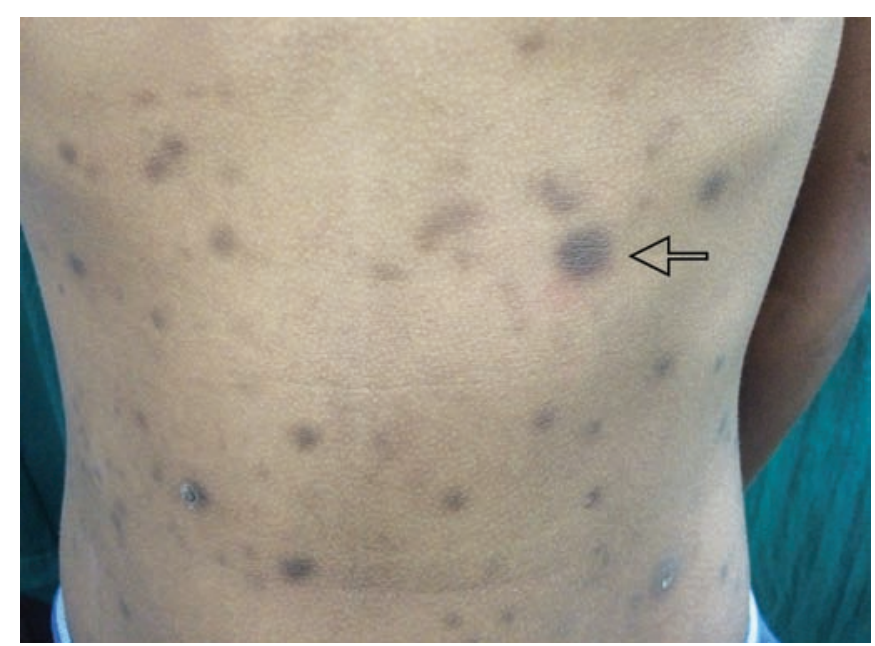

Fig. 2: Accentuated skin markings of lesion as pointed by an arrow

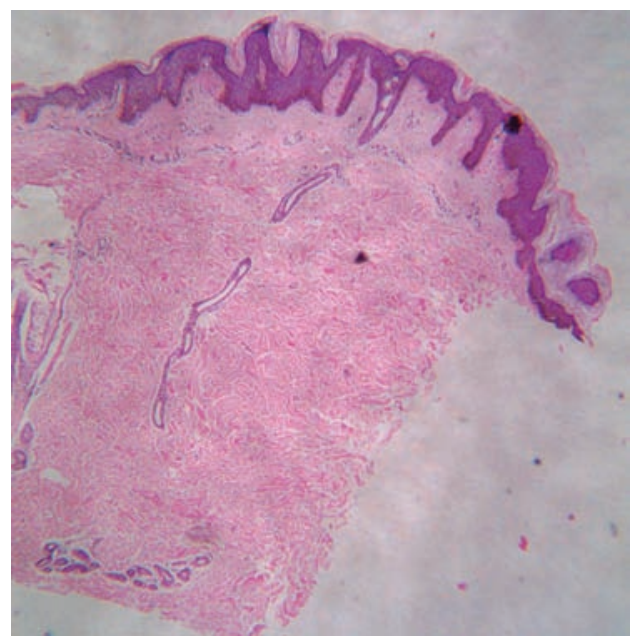

Fig. 4: Hematoxylin and eosin-stained section showing papillomatosis along with hyperkeratotic and orthokeratotic stratum corneum $(4 \times)$

melanin content in the basal layer (Figs 4 and 5). Based on clinicopathological correlation, we made a diagnosis of IEMP. We counseled the patient regarding benign nature of his skin disease.

\section{DISCUSSION}

Idiopathic eruptive macular pigmentation needs to be distinguished from lichen planus pigmentosus, erythema dyschromicum perstans, fixed drug eruption, mastocytosis, and postinflammatory hyperpigmentation. Only with the help of clinicohistopathological corelation, this underreported condition can be diagnosed. Here we would like to highlight an important clinical finding of accentuated skin markings on dermoscopy, which can provide a clinical clue toward diagnosis of IEMP. None of the above differential has similar findings on clinical examination. Dermoscopic evaluation confirmed the accentuated skin marking in lesions of IEMP, which closely resembles acanthosis nigricans. Papillomatosis on

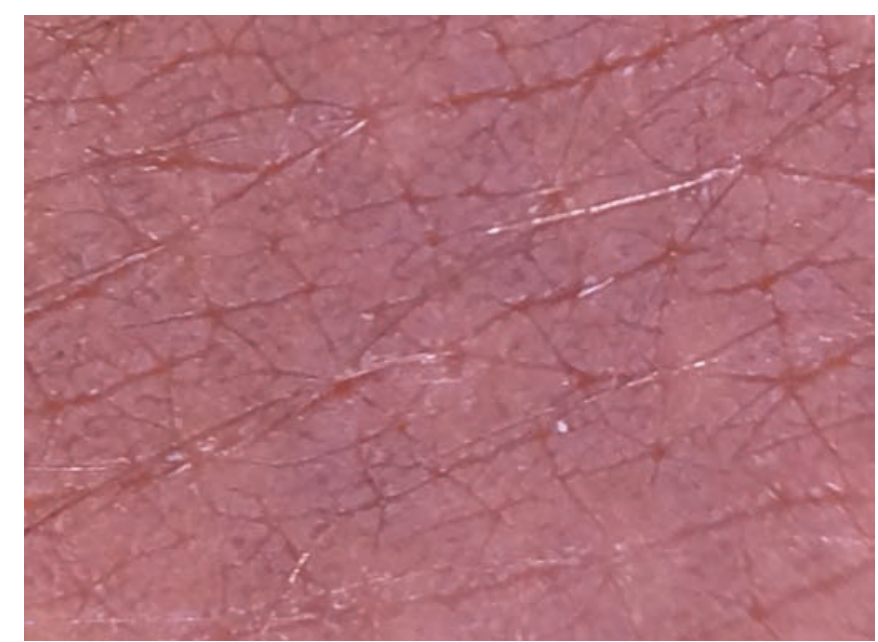

Fig. 3: Contact dermoscopy of lesional skin showing accentuated dermatoglyphics

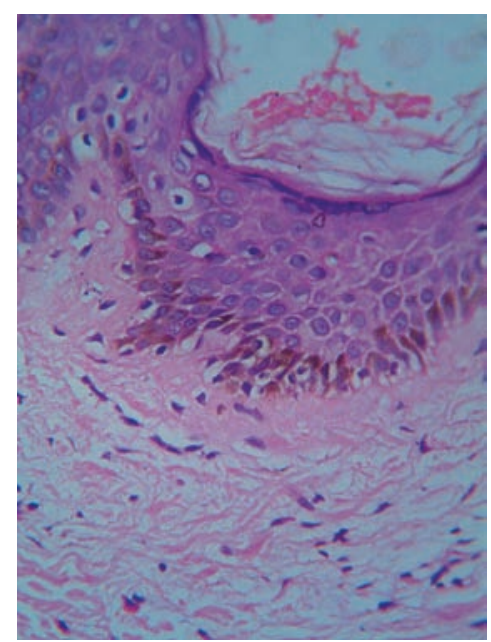

Fig. 5: Hematoxylin and eosin-stained section showing prominent pigmentation of basal keratinocytes (40x)

histology correlates with the exaggerated skin markings on clinical grounds. Joshi et $\mathrm{al}^{3,4}$ and Begum et $\mathrm{al}^{5}$ have proposed that pigmented papillomatosis is a diagnostic histological finding in all cases of IEMP from India. In our case too, we were able to demonstrate pigmented papillomatosis. Verma and Thakur ${ }^{6}$ commented that pigmented papillomatosis needs to be incorporated in the original diagnostic criterion, and we too humbly agree with the suggestion of the authors.

We emphasize that accentuated skin on clinical examination on dermoscopy and pigmented papillomatosis (as proposed by Joshi) can help us in clinching the diagnosis of IEMP.

With the help of adding an additional clinical finding for this entity, underreporting of the disease can be overcome. It will also help in avoiding unnecessary treatment for this self-resolving disease. It is also necessary to make dermatopathologists and pediatricians aware of this condition, so that it will not miss during reporting and making differential diagnosis. 
Idiopathic eruptive macular pigmentation has been most commonly reported in children and adolescents, in whom it is always difficult to do skin biopsy. If dermoscopy findings support the clinical findings, it will be helpful in treating pediatric patients without any trauma. But definitely more case diagnosis with the help of dermoscope is needed to support the findings of this study.

Related with IEMP, one more aspect needs to be explored with the help of research, i.e., whether it is separate entity or related to the development of future acanthosis nigricans in these patients.?

\section{REFERENCES}

1. Degos R, Civatte J, Belaich S. Idiopathic eruptive macular pigmentation (author's transl). Ann Dermatol Venereol 1978 Feb;105(2):177-182.
2. Galdeano SC, Léauté-Labrèze C, Bioulac-Sage P, Nikolic M, Taïeb A. Idiopathic eruptive macular pigmentation: report of five patients. Pediatr Dermatol 1996 Jul;13(4):274-277.

3. Joshi R. Idiopathic eruptive macular pigmentation. Indian J Paediatr Dermatol 2015;16:54-55.

4. Joshi R. Idiopathic eruptive macular pigmentation with papillomatosis: report of nine cases. Indian J Dermatol Venereol Leprol 2007;73(6):402-405.

5. Begum A, Ramachandra BV, Kumar A. Idiopathic eruptive macular pigmentation. Indian J Paediatr Dermatol 2014;15(3):114-116.

6. Verma S, Thakur BK. Idiopathic eruptive macular pigmentation with papillomatosis. Indian Dermatol Online J 2011 Jul-Dec;2(2):101-103.

7. Pang YZ, Koh WL, Ang CC. Idiopathic eruptive macular pigmentation with papillomatosis (IEMPP): a controversial entity. Dermatol Online J 2016 Apr;22(4). doj_30622. Retrieved from: http://escholarship.org/uc/item/2ms041h2 